\title{
Deep imaging of Virgo Z: not a nearby ultrafaint dwarf galaxy, but a distant compact group of galaxies ${ }^{\star}$ (Research Note)
}

\author{
B. Barbuy ${ }^{1}$, E. Bica ${ }^{2}$, S. Ortolani ${ }^{3}$, and A. Lopes-Souza ${ }^{1}$ \\ 1 Universidade de São Paulo, IAG, Rua do Matão 1226, Cidade Universitária, 05508-900 São Paulo, Brazil \\ e-mail: barbuy@astro.iag.usp.br; andre.lopes.souza@usp.br \\ 2 Universidade Federal do Rio Grande do Sul, Departamento de Astronomia, CP 15051, 91501-970 Porto Alegre, Brazil \\ e-mail: bica@if.ufrgs.br \\ 3 Università di Padova, Dipartimento di Fisica e Astronomia Galileo Galilei, Vicolo dell'Osservatorio 2, 35122 Padova, Italy \\ e-mail: sergio.ortolani@unipd.it
}

Received 22 May 2013 / Accepted 3 October 2013

\begin{abstract}
Context. The search for Milky Way faint satellite galaxies and star clusters has been intensified in recent years thanks to the Sloan Digital Sky Survey (SDSS), and other efforts to verify candidate objects.

Aims. We observed the Milky Way satellite candidate Vir Z, reported by Walsh et al. (2009, AJ, 137, 450), from the detection of an overdensity in the SDSS database.

Methods. Deep subarcsec seeing images from the New Technology Telescope (NTT) and Galileo telescopes were obtained in order to infer the nature of the proposed overdensity.

Results. The image and colour-magnitude diagram of the Vir Z field shows no dwarf galaxy. Instead, we identified a compact group of galaxies near the center of the Vir $\mathrm{Z}$ field at redshift $z \approx 0.3 \pm 0.2$, based on the apparent magnitudes of the brightest galaxies assuming $L=L *$, and on angular sizes of the spirals assuming physical sizes equal to M 31
\end{abstract}

Key words. Galaxy: general - galaxies: groups: individual: Virgo Z

\section{Introduction}

From the Sloan Digital Sky Survey (SDSS), at least 17 satellites have been confirmed so far, and most of these are ultra-faint dwarf galaxies (e.g. Belokurov et al. 2007; Muñoz et al. 2012).

To trace faint Milky Way satellite candidates, Walsh et al. (2009) used a detection algorithm and looked for overdensities after filtering the sources in colour-magnitude diagrams (CMDs). Among the SDSS overdensities, Walsh et al. (2009) reported a list of four candidates that might be satellites, including CVn W, Her X, UMa Y, and Vir Z.

The candidate dwarf galaxy in Vir $\mathrm{Z}$ is the subject of the present study. It is located at $\mathrm{J} 2000 \alpha=12^{\mathrm{h}} 20^{\mathrm{m}} 19^{\mathrm{s}}, \delta=$ $-01^{\circ} 21^{\prime} 00^{\prime \prime}$, with galactic coordinates $l=288^{\circ} .35, b=+60^{\circ} .43$ where, according to Walsh et al. (2009), an overdensity of sources in a region of roughly $24^{\prime} \times 12^{\prime}$ is seen to lie at a distance of $40 \mathrm{kpc}$ from the Sun as expected from its CMD. We have been inspecting SDSS candidates for satellite dwarf galaxies, or globular clusters, as well as newly found objects, such as the globular cluster Segue 3 (Ortolani et al. 2013), using the ESO New Technology Telescope (NTT) and INAF Telescopio Nazionale Galileo (TNG) 4m-class telescopes.

In Sect. 2 the observations are described. In Sect. 3 star counts are presented. In Sect. 4 the NTT and TNG images are analysed, with distance and redshift estimated from magnitudes and angular sizes. In Sect. 5 conclusions are drawn.

\footnotetext{
* Observations obtained at the European Southern Observatory, proposal 089.D-0194(A).
}

\section{Observations}

We used the NTT equipped with the EFOSC2 instrument, and ESO CCD \# 40, UV-flooded with MPP technology, and $2048 \times$ 2048 pixels. The pixel size of $15 \mu \mathrm{m}$ corresponds to $0.12^{\prime \prime} /$ pixel. It was read binned in two by two mode. The field of view is $4.1 \times 4$.'1. Twenty Landolt stars were measured in photometric conditions, and used for calibrations. The DAOPHOT II package (Stetson 1987) was employed to measure the instrumental magnitudes. Landolt stars $(1983,1992)$ were used for magnitude calibrations: $V=v+0.04(V-I)+28.51 \pm 0.015 \mathrm{mag}$; $I=i+0.01(V-I)+27.96 \pm 0.015 \mathrm{mag}$, for exposure times of $15 \mathrm{~s}$ and airmasses of 1.15 . The zero point uncertainty is \pm 0.04 . The standard La Silla coefficients for atmospheric extinction using EFOSC2 are $C_{V}=0.16 \mathrm{mag} / \mathrm{airmass}, C_{I}=$ $0.08 \mathrm{mag} / \mathrm{airmass}$. The telescope field central coordinates are $\mathrm{J} 2000 \alpha=12^{\mathrm{h}} 20^{\mathrm{m}} 19^{\mathrm{s}}, \delta=-01^{\circ} 20^{\prime} 50^{\prime \prime}$.

The field of Vir Z was also observed on June 8, 2013, in Johnson-Cousins $V, I$ at the Telescopio Nazionale Galileo (TNG), equipped with the spectrograph/focal reducer imager DOLORES. The camera is equipped with a $2048 \times 2048$ E2V 4240 thinned back-illuminated, Astro-BB coated CCD, with a pixel size of $13.5 \mu \mathrm{m}$ corresponding to 0 '.252/pixel and a field of view of $8.6 \times 88^{\prime} 6$. An offset field was observed at $20^{\prime}$ north, under photometric conditions, and a seeing of $1 \mathrm{arcsec}$. The telescope field and offset field central coordinates are $\mathrm{J} 2000 \alpha=12^{\mathrm{h}} 20^{\mathrm{m}} 19^{\mathrm{s}}, \delta=-01^{\circ} 21^{\prime} 00^{\prime \prime}$, and $\mathrm{J} 2000 \alpha=12^{\mathrm{h}} 20^{\mathrm{m}} 19^{\mathrm{s}}, \delta=-01^{\circ} 01^{\prime} 00^{\prime \prime}$. Details of the reduction procedure are described in Ortolani et al. (2013), and the 
Table 1. Log of observations taken on May 20, 2012 at the NTT, and on June 8, 2013 at the TNG.

\begin{tabular}{|c|c|c|c|c|c|c|c|c|}
\hline \multicolumn{2}{|c|}{ Filter exp. } & Seeing & Filte & er exp. & Seeing & Filter & exp. & $\overline{\text { Seeing }}$ \\
\hline & {$[\mathrm{s}]$} & $(\operatorname{arcsec})$ & & {$[\mathrm{s}]$} & $(\operatorname{arcsec})$ & & [s] & $(\operatorname{arcsec})$ \\
\hline & NTT & & & TNG & & & Offset & \\
\hline$V$ & 60 & 0.5 & V & 1800 & 1.0 & V & 30 & 1.0 \\
\hline$I$ & 30 & 0.5 & $I$ & 30 & 0.8 & V & 30 & 1.0 \\
\hline$V$ & 1800 & 0.65 & $I$ & 1200 & 1.0 & V & 1800 & 1.0 \\
\hline$I$ & 1200 & 1.0 & & & & $I$ & 1200 & 0.9 \\
\hline
\end{tabular}

Notes. Filter, exposure time (s), and seeing $(\operatorname{arcsec})$ are given for the NTT field, and TNG main and offset fields.

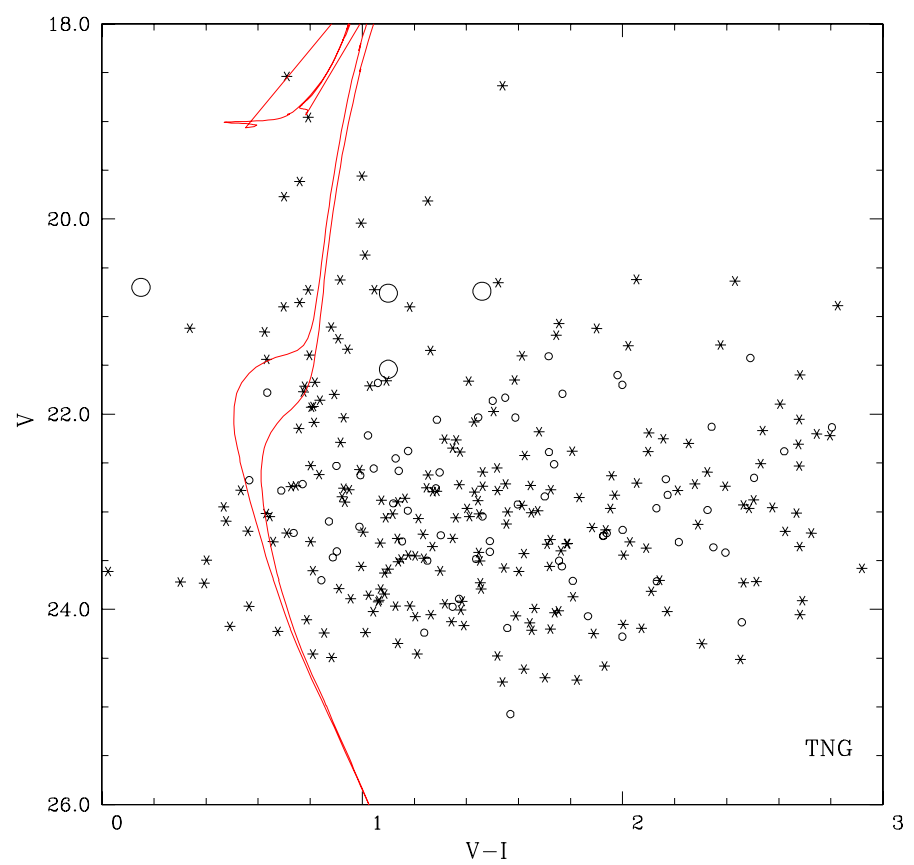

Fig. 1. $V$ vs. $V-I$ CMD corresponding to the TNG full field of the Vir Z object. Large open circles: galaxies of compact group; small open circles: likely field galaxies; stars: stars and distant unresolved galaxies. PARSEC Padova isochrones of 8 and $13.5 \mathrm{Gyr}$ and $[\mathrm{Fe} / \mathrm{H}]=-1.5$, located at a distance of $40 \mathrm{kpc}$, are overplotted.

present calibration equations for $10 \mathrm{~s}$. and airmass of 1.25 are $V=28.46-0.06(V-I)+v \pm 0.015 \mathrm{mag} ; I=28.37-0.15(V-$ $I)+i \pm 0.015 \mathrm{mag}$. Magnitude limits for point source detection at $5 \sigma$ are $V \sim 23.9$ and $I \sim 22.8$ at the TNG, and $V \sim 24.1$ and $I \sim 22.8$ at the NTT.

\section{Star counts}

The NTT $V$ vs. $V-I$ Vir Z CMD is shown in Fig. 1 . We do not see evidence of any tight main sequence, as compared, for example, with that in the SEGUE $3 V$ vs. $V-I$ CMD (Ortolani et al. 2013). Instrumental magnitudes are affected by combined effects due to the atmosphere, detector, and filter. The transformation from the instrumental to the calibrated system, including the colour term, minimizes the differences.

In Fig. 1 we overplot PARSEC (Bressan et al. 2012) isochrones of 8 and $13.5 \mathrm{Gyr}$ (as considered by Walsh et al.) and metallicity $Z=0.005([\mathrm{Fe} / \mathrm{H}] \approx-1.5)$, located at $40 \mathrm{kpc}$, showing that they do not correspond to a stellar population. We note
Table 2. Counts of stars in the direction of the Vir Z field.

\begin{tabular}{|c|c|c|c|c|c|}
\hline \multirow[t]{3}{*}{$V$} & \multicolumn{2}{|c|}{ NTT model } & TNG & Offset & \multirow[t]{2}{*}{ Model } \\
\hline & & & $\mathrm{T} \quad \mathrm{S} \quad \mathrm{G}$ & $\mathrm{T} \quad \mathrm{S} \quad \mathrm{G}$ & \\
\hline & & $4 ! 1$ & $8.6 \times 8.6$ & $8.6 \times 8.6$ & \\
\hline $19-21$ & 9 & 19 & $\begin{array}{lll}16 & 16 & 0\end{array}$ & $\begin{array}{lll}7 & 7 & 0\end{array}$ & 42 \\
\hline $19-22$ & 22 & 13 & $\begin{array}{lll}53 & 44 & 9\end{array}$ & $\begin{array}{lll}47 & 39 & 8\end{array}$ & 70 \\
\hline $19-23$ & 40 & 22 & 15111338 & $\begin{array}{lll}125 & 89 & 36\end{array}$ & 84 \\
\hline $19-24$ & 82 & 31 & 25819464 & $255 \quad 18174$ & 111 \\
\hline
\end{tabular}

Notes. T, S and G correspond to total number of objects, number of stars and galaxies respectively. Model refers to Besançon models.

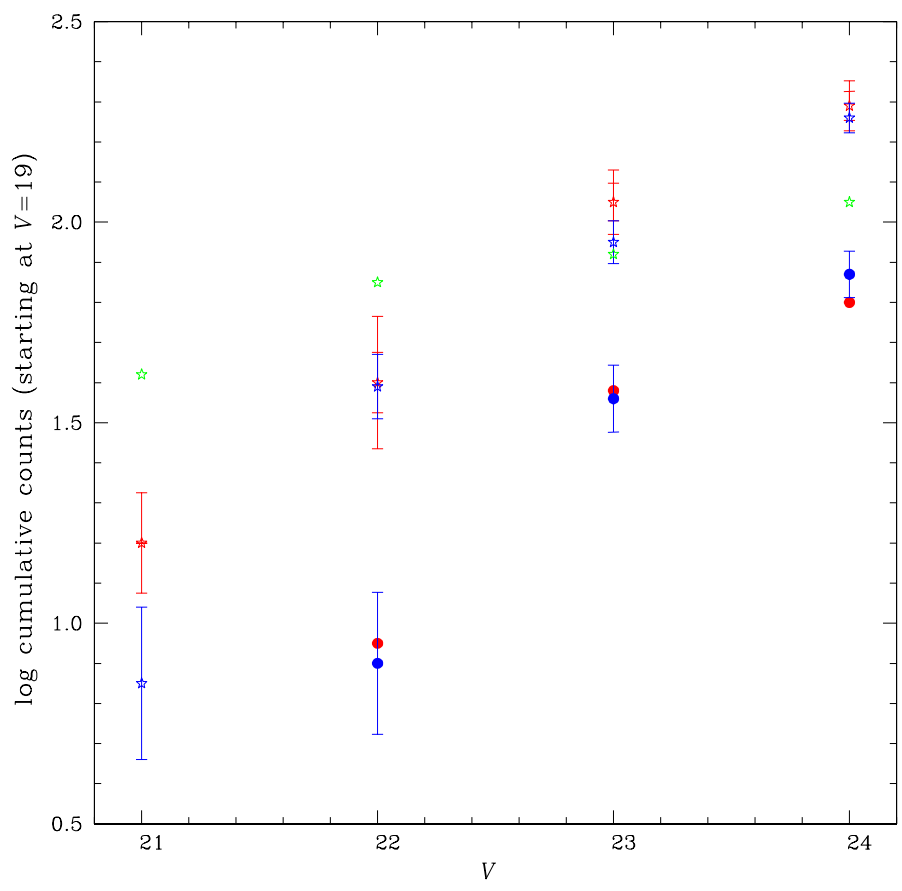

Fig. 2. Cumulative counts of stars and galaxies. TNG field: red; TNG Offset field: blue; symbols: stars: stars; filled circles: galaxies; Besançon model: green stars. Error bars are given for the TNG data points.

that the TNG field encompasses the SDSS Vir Z field, where the overdensity was identified by Walsh et al.

To verify if an overdensity of stars is present in the Vir Z field, a comparison of star counts with model counts of foreground Milky Way stars, and with an offset field, were carried out. We used the Besançon model (Robin et al. 2003) ${ }^{1}$ applied to the fields of view of $4.1 \times 4{ }^{\prime} 1$ (NTT) and 8.6 $\times 8.6(\mathrm{TNG})$ up to a given magnitude limit. In Table 2 are given the counts in the NTT field and Besançon model for the same field, TNG and offset fields, and Besançon models for the TNG area. The DAOPHOT II package is optimized to find stars. Star/galaxy separation was done by checking several thresholds of the sharpness parameter of source shapes, and visually inspecting as well. Therefore, star/galaxy separation is not precise for faint sources, being reliable only up to $V=22.2$. We find that the counts are lower than the Besançon model predictions at bright magnitudes, and higher at faint magnitudes. The count excesses between the observed and Besançon models, for the fainter magnitudes, are very likely due to unresolved galaxies in the CMD. Figure 2 gives the cumulative counts, and suggests that the faint star counts are heavily contaminated by galaxies. To check if it could be due to stars

1 http:model.obs-besancon. fr 
Table 3. Identification of galaxies in the compact group, their Gunn $g$ magnitudes from SDSS, $V$ and $V-I$ from the present NTT observations, the $\mathrm{K}$ correction $k_{V}$, extinction $A_{V}$, and final corrected $V$ magnitudes.

\begin{tabular}{|c|c|c|c|c|c|c|c|c|c|}
\hline ID & SDSS number & $\alpha(\mathbf{J} 2000)$ & $\delta(\mathrm{J} 2000)$ & Gunn $g$ & $V$ & $V-I$ & $k_{V}$ & $A_{V}$ & $V$-corr \\
\hline & & SDSS & & & & NTT & & & \\
\hline galaxy-1 & J122021.00-012034.9 & $12^{\mathrm{h}} 20^{\mathrm{m}} 21^{\mathrm{s}} .0$ & $-01^{\circ} 20^{\prime} 35^{\prime \prime}$ & 21.1 & $20.74 \pm 0.1$ & $1.46 \pm 0.05$ & 0.57 & 0.085 & 20.1 \\
\hline galaxy-2 & $\mathrm{J} 122021.43-012032.9$ & $12^{\mathrm{h}} 20^{\mathrm{m}} 21^{\mathrm{s}} .4$ & $-01^{\circ} 20^{\prime} 33^{\prime \prime}$ & 21.2 & $20.76 \pm 0.1$ & $1.10 \pm 0.05$ & 0.28 & 0.085 & 20.4 \\
\hline galaxy-3 & J122022.03-012036.7 & $12^{\mathrm{h}} 20^{\mathrm{m}} 22^{\mathrm{s}} .0$ & $-01^{\circ} 20^{\prime} 37^{\prime \prime}$ & 20.7 & $20.70 \pm 0.1$ & $0.15 \pm 0.05$ & 0.98 & 0.085 & 19.5 \\
\hline galaxy-4 & J122021.97-012030.4 & $12^{\mathrm{h}} 20^{\mathrm{m}} 22^{\mathrm{s}} .0$ & $-01^{\circ} 20^{\prime} 30^{\prime \prime}$ & 21.8 & $21.54 \pm 0.1$ & $1.10 \pm 0.05$ & 0.28 & 0.085 & 21.2 \\
\hline
\end{tabular}

from an ultrafaint dwarf galaxy or cluster, a comparison with the offset field indicates that the Vir Z vs. its offset field of the TNG observations give essentially the same counts for the magnitude range $19<V<24$ in both fields. The slight excess of stars in the standard field relative to the offset field, at $V<23$ is not confirmed at $V<24$ therefore, the former can be considered a fluctuation. In conclusion, we suggest that a resolved system in the Vir Z field is not detected.

\section{The compact group}

The NTT full field is shown in Fig. 3. There are no obvious signs of a satellite galaxy or a star cluster. On the other hand, we see a group of four relatively bright galaxies at about 0.7 to the NE of the field centre, with coordinates $\mathrm{J} 2000 \alpha=12^{\mathrm{h}} 20^{\mathrm{m}} 22^{\mathrm{s}}, \delta=$ $-01^{\circ} 20^{\prime} 32^{\prime \prime}$.

Figure 4 displays an enlarged view of the galaxy group. The four brighter objects appear to form a galaxy compact group, surrounded by faint or low suface brightness galaxies and stars. This configuration basically reflects the properties of the field described by Walsh et al. (2009). We also observed offset fields $15^{\prime}$ south with the NTT, and $20^{\prime}$ north with the TNG, with same exposure times and seeing conditions, where no effects of an overdensity are seen.

As defined by Hickson $(1982,1997)$, compact groups of galaxies typically have four or five galaxies. The present group appears to satisfy some of the Hickson criteria for defining a compact group: (i) the four galaxies must differ by less than 3 magnitudes; (ii) mean surface brightness $\mu_{\mathrm{V}}<26$ magnitudes $\operatorname{arcsec}^{-2}$ in the POSS-I $E$ band, which is close to $R$; the corresponding limit in $V$ should be numerically larger, since galaxies have positive $V-R$. We estimated $\mu_{\mathrm{V}} \approx 25.7$ magnitudes $\operatorname{arcsec}^{-2}$, therefore fulfilling this criterion; and (iii) the group appears to be isolated, as seen in Fig. 3, and from the SDSS overdensity shown by Walsh et al. (2009) in their Fig. 10. In Fig. 4 we overplot the smallest circumscribed circle, as well as a concentric circle of 3 times its angular radius, displaying the isolation ring, in which a typical Hickson group should have no galaxies.

Table 3 condenses information from the SDSS catalog for the four galaxies. We report $V$ magnitudes and $V-I$ colours, measured with an aperture of diameter 2.'5. It is important to note that our observations were taken with a $3.5 \mathrm{~m}$ telescope (NTT) under exceptional seeing of 0.'5 (Table 1) therefore, the present observations are considerably deeper than those from the SDSS database. We have applied K-corrections, using the calibrations by Chilingarian \& Zolotukhin $(2012)^{2}$ with values reported in Table 3 . Foreground and internal reddening have to be added to the K-correction. Foreground reddening is estimated to be of $A_{V}=0.070$ from Schlafly \& Finkbeiner (2011), and $A_{V}=0.085$ from Schlegel et al. (1998); with $R=3.2$ we get

\footnotetext{
2 http://kcor.sai.msu.ru
}

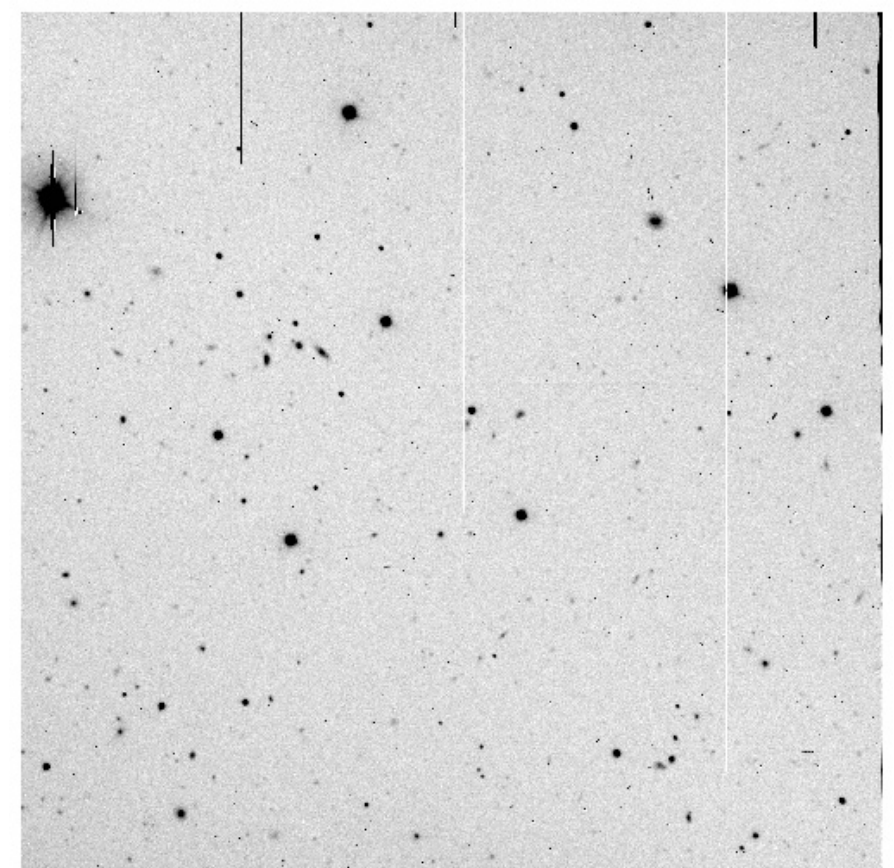

Fig. 3. Vir Z: NTT full field of $4.1 \times 4$.'1, obtained with a $30 \mathrm{~min}$. exposure in $V$. North is to the top and east to the left.

$E(B-V)=0.022$ or 0.027 , respectively. We do not correct for internal reddening, as our group is sufficiently close $(z \approx 0.3)$ that it is unlikely to be a dusty starburst. The $V$-corrected magnitudes are given in Table 3.

A comparison of the SDSS Gunn $g$ and present $V$ magnitudes shows a very good agreement, noting that galaxy 2 is somewhat affected by a faint companion. Galaxy 3 is bluer than the group companions (Table 3 ). This is possibly a merging galaxy, owing to its shape and faint structures nearby.

\subsection{Distance and redshift determination}

The luminosity distance can be derived using the apparent and absolute magnitude values: $m-M=-5+5 \log \left(d_{\mathrm{L}}(\mathrm{pc})\right)$. For the sample galaxies we have a mean magnitude of $V \sim 20.3$. For a typical absolute visual magnitude, we estimated $M_{V}$ as follows. Based on a SDSS galaxy catalog, Blanton et al. (2003) derived characteristic luminosities, as defined by Schechter (1976), of $M_{g}^{*}=-19.39+5 \log h=-20.25$ and $M_{r}^{*}=-20.44+5 \log h=$ -21.3. Adopting $V=g-0.03-0.42(g-r)$ (Windhorst et al. 1991), we get $M_{V}^{*}=-20.72$. We therefore adopt a typical absolute visual magnitude of $M_{V}=-20.7$. We then obtain $d_{\mathrm{L}}=1.58 \mathrm{Gpc}$. In order to derive the redshift $z$, we employed the relation for the luminosity distance of a galaxy as a function of 


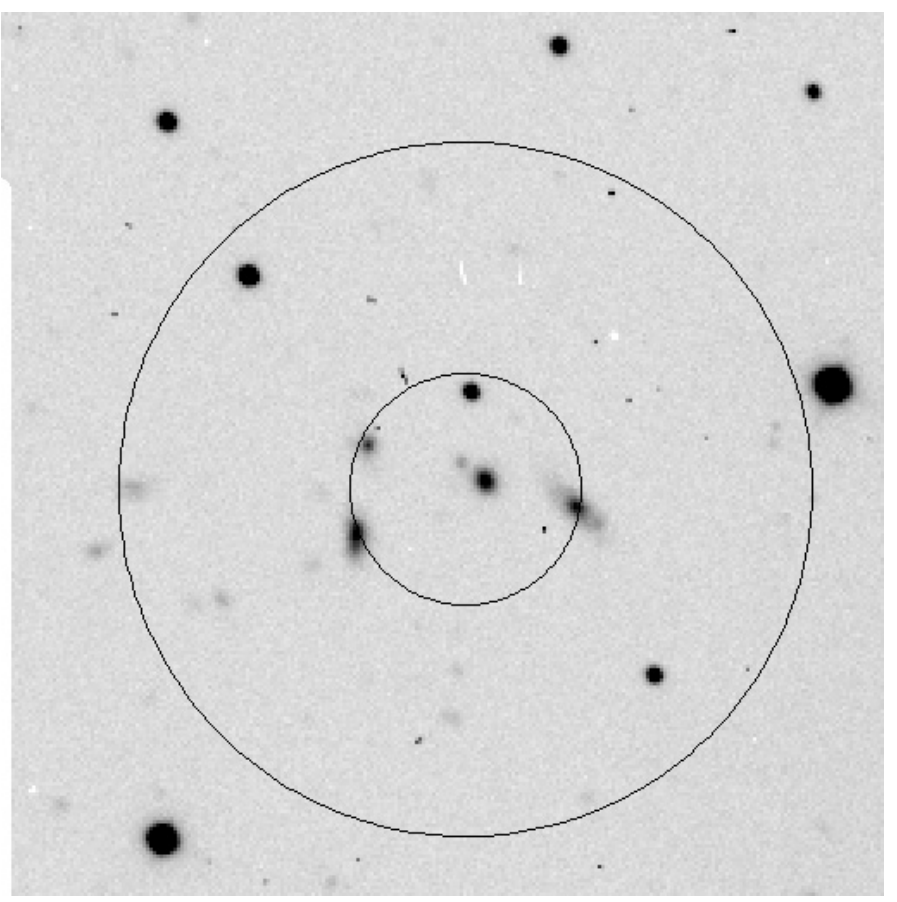

Fig. 4. Enlargement of Fig. $1\left(1^{\prime} \times 1^{\prime}\right)$ containing the galaxy group, and identification of galaxies (Table 3 ).

redshift given by Wickramasinghe \& Ukwatta (2010). Adopting the Hubble constant of $H_{0}=67.3 \mathrm{~km} \mathrm{~s}^{-1} \mathrm{Mpc}^{-1}$ recently obtained from the Planck Surveyor mission (Planck Collaboration 2013), together with a flat Universe with dark energy density parameter $\Omega_{\Lambda}=0.685_{-0.018}^{+0.016}$, and matter density parameter $\Omega_{\mathrm{m}}^{0}=$ 0.315 , we get a redshift of $z \approx 0.30$.

We can also estimate the redshift from the angular diameter of a galaxy (e.g. de Souza 2004), through the formula $\Theta=D / d_{\mathrm{A}}(z)$, where $D$ is the intrinsic diameter of a typical luminous galaxy. We have measured angular sizes of $8^{\prime \prime}$ for the two larger elongated and probably spiral galaxies 1 and 3. For completeness, we measured the FWHM of light profiles resulting to be around 9.' $2 \pm 0.2$ pixels at the TNG image, or 2.3 for the larger galaxies. The diameter of the Milky Way is estimated to be of $D \sim 31-40 \mathrm{kpc}$, and that of Andromeda is of $\sim 40 \mathrm{kpc}$; we assume a true diameter of $36 \mathrm{kpc}$, which leads to a redshift of $z=0.32$.

The uncertainties in distance and redshift are estimated by varying the magnitudes and sizes, as follows: a) a measured error in apparent magnitude of \pm 0.1 mag gives uncertainties of \pm 0.01 in redshift and ${ }_{-0.11}^{+0.17}$ in distance; b) the uncertainty in absolute magnitude adopted is $V=-20.7 \pm 0.6$, resulting in $d=1.59_{-0.4}^{+0.5} \mathrm{Gpc}$, and $z=0.30 \pm 0.04$ in redshift. From a) and b) we get an uncertainty of $z=0.30 \pm 0.04$ in redshift and $d=1.59_{-0.42}^{+0.53} \mathrm{Gpc}$ in distance; c) measured error in angular size is $1^{\prime \prime}$ leading to an uncertainty of ${ }_{-0.05}^{+0.06}$ in redshift; and d) a true diameter of $36 \pm 4 \mathrm{kpc}$ leads to a redshift of $z=0.32_{-0.05}^{+0.07}$. From c) and d) we get a redshift uncertainty of of $z=0.32_{-0.07}^{+0.09}$. In conclusion, from the magnitude formula, we get $z=0.30 \pm 0.04$ and distance $d=1.59_{-0.42}^{+0.53} \mathrm{Gpc}$, and from the angular size formula we also get $z=0.32_{-0.07}^{+0.09}$. A mean of results from the two formulae, plus internal error, leads to a redshift of $z=0.31_{-0.18}^{+0.19}$.

\section{Conclusions}

We observed and analysed the properties of Vir Z, a satellite candidate detected in the SDSS survey, as suggested by Walsh et al. (2009). We obtained images with the NTT and TNG telescopes, thus larger than the SDSS telescope, and under very good seeing conditions. Deep images allowed us to identify a compact group of galaxies located at $0.7^{\prime}$ north-east of the suggested overdensity centre. We do not confirm a dwarf galaxy or a stellar cluster in the area. As a check, we produced a $V$ vs. $V-I$ diagram, and no evolutionary sequences corresponding to any stellar systems is present. From inspection of the offset field, and the larger field of view towards the Vir Z target taken with the TNG, compared with the NTT field, we can conclude that there does not seem to be any other conspicuous feature, such as a resolved dwarf galaxy or a star cluster.

We detected instead a galaxy group that obeys the main Hickson (1982) criteria for defining a compact group. There is good agreement between the Gunn $g$ SDSS and our $V$ magnitudes, and there is little spread in magnitude difference among the four galaxies forming the compact group. Galaxy 3 is considerably bluer than the others, and from its morphology and outer structures, it might be a merging system. Compact groups of galaxies are expected at intermediate and high redshifts (e.g., de Carvalho et al. 2005), so that our main contribution in this paper is the non-detection of a dwarf galaxy corresponding to the Vir Z object.

In conclusion, our results do not favour a stellar overdensity in terms of an extended ultrafaint dwarf galaxy. We interpret the SDSS overdensity as a plain mixture of faint stars and galaxies. The extended distribution of faint galaxies may include one or more faint galaxy clusters. The present galaxy group appears to be a small structure near the peak of the SDSS overdensity, and could be the reason for the indication of Vir $\mathrm{Z}$ as a candidate dwarf galaxy.

Acknowledgements. We are grateful to Gary Mamon for very useful comments that greatly improved this Note. B.B. and E.B. acknowledge grants from the Brazilian agencies CNPq and Fapesp. S.O. acknowledges the Italian Ministero dell'Università e della Ricerca Scientifica e Tecnologica.

\section{References}

Belokurov, V., Zucker, D., Evans, N., et al. 2007, ApJ, 654, 897 Blanton, M. R., Hogg, D. W., Bahcall, N. A., et al. 2003, ApJ, 592, 819 Bressan, A., Marigo, P., Girardi, L., et al. 2012, MNRAS, 427, 127 Chilingarian, I., \& Zolotukhin, I. 2012, MNRAS, 419, 1727

de Carvalho, R. R., Gonçalves, T. S., Iovino, A., et al. 2005, AJ, 130, 425 de Souza, R. E. 2004, Introdução à Cosmologia (EDUSP)

Hickson, P. 1982, ApJ, 255, 382

Hickson, P. 1997, ARA\&A, 35, 357

Landolt, A. U. 1983, AJ, 88, 439

Landolt, A. U. 1992, AJ, 104, 340

Muñoz, R., Geha, M., Côté, P., et al. 2012, ApJ, 753, L15

Ortolani, S., Bica, E., \& Barbuy, B. 2013, MNRAS, 433, 1966

Planck Collaboration 2013, A\&A, submitted [arXiv: 1303. 5076]

Robin, A., Reylé, C., Derrièrre, S., \& Picaud, S. 2003, A\&A, 409, 523

Schechter, P. 1976, ApJ, 203, 297

Schlafly, E. F., \& Finkbeiner, D. P. 2011, ApJ, 737, 103

Schlegel, D. J., Finkbeiner, D. P., \& Davis, M. 1998, ApJ, 500, 525

Stetson, P. B. 1987, PASP, 99, 191

Walsh, S., Willman, B., \& Jerjen, H. 2009, AJ, 137, 450

Wickramasinghe, T., \& Ukwatta, T. N. 2010, MNRAS, 406, 548

Windhorst, R. W. 1991, ApJ, 380, 362 